\title{
EFECTO RAPIDO DE $\alpha$-TOCOFEROL SOBRE LA OXIDACION DE LDL DE CONEJOS HIPERCOLESTEROLEMICOS
}

\author{
Lidia Cruz Neyra ${ }^{1,2}$, \\ Paulo Afonso Ribeiro Jorge ${ }^{3}$ \\ Regina Michico Ozaki ${ }^{3}$ \\ Admar Costa de Oliveira ${ }^{2}$.
}

Facultad de Ciencias Biológicas, Universidad Ricardo Palma' Laboratorio de Lípidos, $\mathrm{FEA}^{2}$, Laboratorio experimental de Cardiología, $\mathrm{FCN}^{3}$, Universidad de Campinas, São Paulo, Brazil

\section{RESUMEN}

Diferentes líneas de evidencias sugieren que la modificación oxidativa de la lipoproteína de baja densidad (LDL) es aterogénica y los antioxidantes pueden evitar su alteración oxidativa. El propósito de este trabajo fue estudiar los efectos de $\alpha$-Tocoferol (vitamina E), en periodos cortos, sobre los productos de la peroxidación de lípidos de LDL, como es el Malondialdehído (MDA) y sobre el perfil lipídico en conejos hipercolesterolémicos.

El experimento consistió de cuatro grupos con ocho conejos, machos, blancos, de raza New Zealand y con un peso promedio de $1.8 \pm 0.2 \mathrm{Kg}$, cada grupo. El grupo hipercolesterolémico $(\mathrm{HH})$ recibió una dieta con $5 \%$ de colesterol y $10 \%$ de aceite de coco $(\mathrm{w} / \mathrm{w})$ por 30 días, los otros tres grupos recibieron la misma dieta con un suplemento de 50 U.I. de $\alpha$-Tocoferol administrado oralmente a través de una sonda gástrica, a los 2 (E-2), 4 (E-4) y 6 (E-6) días últimos del periodo experimental.

Fueron determinados los niveles plasmáticos de colesterol total, colesterol-LDL, colesterol-VLDL, colesterol-HDL, triacilglicéridos y la concentración de MDA en las partículas de LDL nativa y oxidada.

Los niveles de MDA fueron significativamente reducidos en las partículas de LDL nativa y oxidada ( $46 \%$ y $36 \%$ respectivamente), que procedían de los animales tratados con $\alpha$ tocoferol, durante dos días.

Los niveles plasmáticos de colesterol total, colesterol-LDL y triacilglicéridos fueron disminuidos en el grupo que recibió $\alpha$-tocoferol durante 6 días $(\mathrm{p}<0.05)$. No se observó diferencia significativa en los niveles de colesterol-HDL. La composición química de LDL de los grupos HH, E-2, E-4 y E-6 no presentó diferencia significativa.

El $\alpha$-Tocoferol, rápidamente fue capaz de disminuir los niveles de MDA en las partículas de LDL y c-LDL, sugiriendo un potencial efecto antiaterogénico.

Palabras Claves: $\alpha$-Tocoferol, Lipoproteína de baja densidad (LDL), Hipercolesterolemia

\section{SUMMARY}

Rapid Effect of $\alpha$-Tocopherol on the LDL oxidation from Hypercholesterolemic Rabbits: Several lines of evidence suggest that oxidatively modified low density lipoprotein (LDL) is atherogenic and that antioxidants may protect LDL against oxidation.

The purpose of this work was to study the time course of the vitamin E on the lipid peroxidation product malondialdehyde (MDA) of LDL and the blood lipid profile in hypercholesterolemic rabbits. Male New Zealand White rabbits $(1.8 \pm 0.2 \mathrm{Kg}$ weight $)$ were divided into four groups ( 8 rabbits/group). The hypercholesterolemic group ( $\mathrm{HH}$, control) received a diet enriched with cholesterol $(5 \% \mathrm{w} / \mathrm{w})$ and coconut oil $(10 \% \mathrm{w} / \mathrm{w})$ for 30 days and 
the others groups received the same diet plus 50 I.U. of $\alpha$-Tocopherol administered orally once a day by gavage, for the last 2 (E-2), 4 (E-4) and 6 (E-6) days of the experiment.

The plasma concentration of total cholesterol, LDL-cholesterol, VLDL-cholesterol, HDL-cholesterol, triglycerides and MDA of native and oxidized LDL were measured.

The MDA level was significantly reduced in native and oxidized LDL $(46 \%$ and $36 \%$ respectively) in the animals treated with vitamin E for two days. Total cholesterol, LDL-c and triglycerides were decreased in the group that received $\alpha$-tocopherol for 6 days $(p<0.05)$. No significant statistical differences were found in the cholesterol-HDL levels. The chemical composition of the LDL from the HH, E-2, E-4 and E-6 groups were not significant.

$\alpha$-Tocopherol was rapidly able to decrease the MDA and c-LDL levels and thus suggesting thus a potentially anti-atherosclerotic property.

\section{Key Words: $\alpha$-Tocopherol, low density lipoprotein $(L D L)$, hypercholesterolemia}

\section{INTRODUCCION}

En recientes investigaciones, se ha reportado que las vitaminas antioxidantes, incluyendo a la vitamina $\mathrm{E}$, beta-caroteno $\mathrm{y}$ vitamina $C$ pueden reducir el riesgo de enfermedades cardionarias, recibiendo considerable atención por la comunidad científica y público en general.

Algunas evidencias sugieren que la lipoproteína plasmática de baja densidad (Low Density Lipoprotein, LDL), modificada oxidativamente juega un rol importante en el desarrollo de la aterosclerosis, debido a su captura vía receptores secuestrantes de macrófagos, generando la formación de células espumosas (KANNEL et al., 1979; STEINBERG et al., 1989; AVIRAM, 1993; WITZUM, 1993; DAVIES \& WOOLF, 1993).

En la hipercolesterolemia, las concentraciones plasmáticas de LDL incrementan y las lipoproteínas permanecen más tiempo en circulación, que permiten mayor susceptibilidad a la modificación oxidativa. La oxidación de LDL (ox-LDL) se produce por ataque de radicales libres, formándose productos de peroxidación (HEINECKE et al., 1987). En la oxidación, también, se produce derivados de los epsilon-amino de los residuos de lisina que se presentan en la apoproteína B (apo-B), proteína que forma parte del complejo de la LDL (STEINBRECHER et al., 1987). Los productos de la peroxidación que generan estos derivados son el malondialdehído (MDA) y el 4-hidroxinonenal (4-HNE), entre otros (ESTERBAUER et al., 1987)
Anticuerpos específicos contra los epitopes de la LDL oxidada reconocen este material en las lesiones ateroscleróticas pero no en las arterias normales (HABERLAND et al., 1988; BOYSS et al., 1989). Se ha reportado que estos anticuerpos se encuentran en el plasma de pacientes con la enfermedad (PALINSKI et al., 1989).

Los antioxidantes tales como el probucol y el hidroxi-butil tolueno (BHT) pueden inhibir el desarrollo de placas aterogénicas en conejos genéticamente hipercolesterolemicos, como es el caso de los conejos Watanabe (WHHL) y conejos alimentados con dietas ricas en colesterol (KANNEL et al., 1979, KITA et al 1987, DAVIES et al., 1989). Sin embargo, el uso de estos agentes por humanos, para prevenir la aterosclerosis, es limitado (BUCLEY at al., 1989; HIROSE et al., 1981).

Por otro lado, la peroxidación de las partículas de LDL sólo puede ser iniciada cuando existe niveles muy bajos de antioxidantes endógenos. El primer antioxidante lipofilico es el alfa-tocopherol (vitamina E) y el beta caroteno es el último de la línea de defensa contra la peroxidación de la LDL (ESTERBAUER et al., 1987; JIALAL \& GRUNDY, 1992; JIALAL et al., 1995).

El $\alpha$-tocopherol, es el más activo y abundante de la familia de isómeros de la vitamina $\mathrm{E}$, que impide la reacción en cadena de la peroxidación de LDL en el plasma y tejidos (INGOL et al., 1987; MACLIN, 1998)

En un estudio de dieciséis poblaciones europeas, se reporto que existía una significativa correlación inversa entre 
los niveles de $\alpha$-tocoferol y la mortalidad por enfermedad cardiocoronaria (GEY et al., 1991) y el riesgo de angina de pecho (RIEMERSNA et al., 1991).

DIEBER-ROTHENDER et al., 1991 reportaron un aumento de la resistencia a la oxidación de LDL de pacientes que recibieron suplementación de vitamina $\mathrm{E}$ por tres semanas. En animales de experimentación se ha observado que una dieta rica en vitamina $E$ retarda el desarrollo de aterosclerosis inducida por dieta (JANERO, 1991)

El presente estudio tiene como objetivo determinar el efecto en periodos cortos de suplementación de $\alpha$-tocoferol sobre los productos de peroxidación, malonaldehído, (MDA) y perfil lípidico, en conejos hipercolesterolemicos.

\section{MATERIAL Y METODOS}

\section{Materiales}

El colesterol fue obtenido de Amresco, Solon - Ohio (USA). La vitamina $\mathrm{E}$, en su forma acetato de dl-alfa tocoferol fue donado por el laboratorio Ache, el ácido 2-tiobarbitúrico fue obtenido de Sigma Chemical Co. Los Kits para la determinación de colesterol, triaciglicéridos y fosfolípidos fueron proporcionados por Boehringer Mannheim Biochemical (FRG).

\section{Animales y dietas}

Los experimentos fueron siguiendo la guía de cuidados y uso de animales de laboratorio, propuesta por US National Institutes of Health (NIH publication No. 85-23, 1985).

Se usaron treinta y dos conejos, machos blancos, de 12 semanas de edad, de la raza New Zealand, con un promedio de peso corporal de $1.8 \pm 0.2 \mathrm{~kg}$. Los animales fueron asignados al azar para formar cuatro grupos con ocho unidades experimentales en cada uno, con la siguiente asignación: 1. Hipercolesterolémico, grupo control $(\mathrm{HH})$, 2. vitamina E-2, (E-2) 3. Vitamina E-4 (E4) y Vitamina E-6 (E-6).

Todos los animales recibieron una dieta suplementada con $0.5 \%$ de colesterol y $10 \%$ de aceite de coco, por 30 días. Los grupos de Vitamina $\mathrm{E}$ recibieron 50 U.I. diarias de dl- $\alpha$-tocoierol, por vía oral, usando una sonda gástrica, por dos, cuatro y seis días antes de finalizar los 30 días del experimento.

Los animales fueron colocados en jaulas individuales, bajo las mismas condiciones, y proporcionándoles $100 \mathrm{~g}$ de alimento por día, dejando el agua ad-libitum.

\section{Lípidos Plasmáticos}

A los treinta días del experimento, se realizó la punción cardíaca para obtener el plasma sanguíneo de los conejos. En el plasma obtenido se determinaron las concentraciones de lípidos y lipoproteínas y se procedió al aislamiento de la LDL.

La sangre fue colectada en tubos conteniendo EDTA (1 mg/ml), luego el plasma fue separado por una centrifugación suave a $4^{\circ} \mathrm{C}$. El colesterol y triacilglicéridos fueron determinados mediante el uso de Kit enzimático. HDL fue determinada por procedimiento enzimático, después de la precipitación de las lipoproteínas que contienen apoproteína $\mathrm{B}$, usando el reactivo precipitante de Boehringer Mannheim que contiene ácido fosfotúngstico e iones de magnesio.

\section{Aislamiento de LDL}

LDL fue aislada de plasma fresco por ultracentifugación en gradiente de densidad discontinuo, según Havel et al., (HAVEL et al., 1955). El plasma fue centrifugado por $40,000 \mathrm{rpm}$ for 20 hours at $4^{\circ} \mathrm{C}$ en rotor $50.3 \mathrm{~T} 1$ rotor (Beckman L8, Palo Alto, Ca), luego de la centrifugación fue recolectado en la capa superior VLDL $(\mathrm{d}<1.006 \mathrm{~g} / \mathrm{ml})$. El sobrenadante restante fue ajustado a la densidad de 1.006-1.063 $\mathrm{g} / \mathrm{ml}$ usando $\mathrm{KBr} \quad \mathrm{y}$ inuevamente centrifugado por $40,000 \mathrm{rpm}$ por 20 horas Las partículas de LDL aisladas fueron sometidas a diálisis contra buffer fosfato salino PBS, a pH 7.4 a $4^{\circ} \mathrm{C}$ por 24 horas.

La concentración de proteína fue determinada por el método de Lowry et al. (LOWRY et al., 1951), usando suero albúmina de bovino. 


\section{Oxidación de LDL}

LDL $(100 \mu \mathrm{g}$ de proteína/ml de PBS $10 \mathrm{mM}, \mathrm{pH}$ 7.4) fue incubada por 24 horas a $37^{\circ} \mathrm{C}$ con sulfato de cobre $5 \mu \mathrm{M}$ (PARTHASARATHY et al., 1990). El contenido de MDA de LDL oxidada y LDL no oxidada fue medido por el ensayo de Buege y Aust (BUEGE et al., 1978), determinando las Substancias Reactivas con el Acido Tiobarbitúrico (TBARS). La actividad de TBARS fue expresada por la concentración de MDA equivalentes (nmol/mg de proteína de LDL) usando como estándar el 1,1,3,3 tetramethoxypropane, preparado en fresco.

\section{Análisis estadístico}

Todos los resultados fueron expresados como la media \pm desvío estándar (SD). Las comparaciones entre los grupos se realizaron mediante el uso del test de Kruskal-Wallis, siendo el nivel de significancia a $\mathrm{p}<0.05$.

\section{RESULTADOS}

Al finalizar el experimento, al cabo de treinta días, no se observo diferencias significativas en los pesos corporales de los conejos. Los pesos fueron $2.7 \pm 0.2,2.8 \pm$ $0.3,2.8 \pm 0.2$ y $3.0 \pm 0.2$ kilogramos para los grupos $\mathrm{HH}$, vitamina E-2, E-4 y el grupo E-6, respectivamente.

La suplementación de $\alpha$ Tocopherol tuvo un rápido y significativo efecto sobre los lípidos y lipoproteínas plasmáticas de los conejos hipercolesterolémicos, tratados con $\alpha$ tocoferol durante 6 días (E-6).

El colesterol total y triacilglicéridos presentaron diferencias significativas entre el grupo HH (control) y el grupo E-6 (Fig. 1).

Una significante reducción fue observada en los niveles de colesterol-LDL después de seis días de administración de $\alpha$-tocoferol ( $\mathrm{p}<0.05$; tabla 1$)$. No existen diferencias significativas en los niveles de colesterol-HDL entre los grupos control y sometidos a tratamiento $\operatorname{con} \alpha$-tocoferol.
El contenido de MDA en las partículas de LDL nativa y LDL oxidada (nn-LDL and ox-LDL) fueron significantemente reducidas después de dos días de suplementación con $\alpha$-tocoferol ( $\mathrm{p}<0.05 ;$ Fig.2).

En la tabla 2 se puede apreciar la composición en porcentaje de LDL: colesterol triacilglicéridos, fosfolípidos y proteínas; como se observa no existen diferencias significativas en la composición de LDL en los diferentes grupos. $\alpha$ Tocoferol no altera la composición de LDL en conejos hipercolesterolémicos.

\section{DISCUSIÓN}

Muchos autores han sugerido que la modificación oxidativa de LDL tendría un papel clave en el inicio de la aterogenesis, de manera que mecanismos que puedan reducir los niveles de oxidación de LDL se consideran ser antiaterogénicos.

$\mathrm{La}$ inhibición de la modificación oxidativa de LDL en respuesta a cortos tratamientos con $\alpha$-tocoferol no ha sido reportado por la literatura. En el presente estudio, en el cual conejos hipercolestrolémicos son tratados con 50 U.I de dl- $\alpha$-tocoferol, se ha observado una reducción de los productos de peroxidación, como es el MDA en LDL nativa y LDL oxidada, obteniéndose valores muy significativos de $46 \%$ y $36 \%$ respectivamente, después de un tratamiento de dos días de administración de $\alpha$ tocoferol y $65 \%$ y $70 \%$ respectivamente, después de seis días de administración de $\alpha$-tocoferol.

TRABER \& KAYDEN, 1980 reportaron que sujetos normales con dietas suplementadas con vitamina $\mathrm{E}$, sus nivels plasmáticos de $\alpha$ - tocoferol aumentaron en más de un tercio, después de 24 horas. El contenido de $\alpha$-tocoferol en la fracción de quilomicrones y VLDL alcanza un pico después de 4 horas; mientras que la fracción de LDL alcanza un pico a las 24 horas. Conociendo que $\alpha$-tocoferol es incorporado en las partículas dentro de las 24 horas, una disminución de la oxidación puede haber alcanzado al segundo día de la administración de $\alpha$-tocoferol. 
La vitamina $\mathrm{E}$ es conocida ser un agente eficientemente antioxidante, el cual protege a las membranas celulares contra la peroxidación de sus lípidos, debido a que rompe la cadena de reacciones de oxidación de lípidos.

Se ha demostrado que $\alpha$-tocoferol, el cual se presenta en considerables cantidades en las partículas de LDL, particularmente en mayores concentraciones que las membranas celulares, es también un factor importante en la protección contra el ataque de la peroxidación de lípidos de los ácidos grasos polinstarurados de LDL, (ESTERBAUER et al., 1989). Estimando las concentraciones molares de $\alpha$-tocoferol, se concluye que es el principal antioxidante de LDL.

La suplementación con vitamina $\mathrm{E}$ incrementa el contenido de $\alpha$-tocoferol y disminuye la susceptibilidad a la peroxidación de lípidos de LDL catalizada por iones de cobre, $\mathrm{Cu}^{2+}$. (DUTHIE, 1991; HAELLIWELL \& CHIRICO, 1993; HENEKENS, 1998)

En este estudio, el colesterol total, el colesterol-LDL y el contenido de triacilglicéridos fueron reducidos a los seis días de administración de vitamina $E$. WESTROPE et al., 1982 observaron que las concentraciones plasmáticas de colesterol fueron significativamente menores y las lesiones ateroscleróticas disminuidas en grupos que se alimentaban con $1 \%$ de vitamina $\mathrm{E}$ en la dieta, cuando comparadas con un grupo hipercolesterolemicos

QIAO et al,, 1993 observaron que administrando vitamina $\mathrm{E}$ a cuyes con dietas ricas con colesterol, producía una reducción del $12.2 \%$ en los niveles de colesterol total, en un mes. PRASAD \& KALRA, 1993 reportaron que la vitamina $E$ producía una disminución de triacilglicéridos plasmáticos, pero no se presentaba cambios significativos en las concentraciones de colesterol total, colesterol-HDL, colesterol-LDL y colesterol-VLDL.

Por otro lado, WILLIAMS et al., 1992 han demostrado propiedades antihipercolesterolémicos, antioxidantes y antiateroscleróticos de la vitamina $\mathrm{E}$ en conejos genéticamente deficientes del receptor de LDL.

Aunque muchos de las publicaciones científicas están de acuerdo con indicar que $\alpha$-tocoferol protege a las partículas de LDL contra la oxidación, hay divergencia sobre su efecto en los niveles de colesterol plasmáticos. Estudios en conejos con tratamiento de dieta hipercolesterolémica y suplementados con vitamina $\mathrm{E}$ han obtenido resultados divergentes y queda en discusión la habilidad de la vitamina E sobre su intervención en la formación de la placa aterogénica.e inhibir la lesión, es decir se discute su potencia hipocolesterolémica (WESTROPE et al., 1982; GODFRIED et al., 1989; WILLIAMS et al., 1992). Estas discrepancias están aparentemente relacionadas a las diferentes dosis de administración de la vitamina $\mathrm{E}$.

KLEINVELD et al., 1998 reportaron una dosis dietária óptima de vitamina $\mathrm{E}$, son aproximadamente el $0.025 \%(w / w)$. Con esta dosis, los autores encontraron un fuerte efecto antioxidante de la vitamina $\mathrm{E}$ y una mayor reducción de las lesiones ateroscleróticas en la aorta de conejos Watanabe, con hiperlipidemia genética, pero no disminuía los niveles plasmáticos de colesterol total.

De otro lado, se ha reportado que $\alpha$-tocoferol tiene afinidad por los receptores hepáticos para LDL (QURESHI et al., 1986) lo cual puede influir sobre los niveles de colesterol y LDL plasmáticos.

En nuestro estudio, el efecto de $\alpha$ tocoferol está relacionado primariamente a su efecto antioxidante sobre la medida de susceptibilidad a modificación oxidativa de LDL y subsecuentemente su efecto sobre la reducción de los niveles plasmáticos de colesterol (RIBEIRO-JORGE et al., 1998).

Una reducción del colesterol total plasmático en animales tratados con vitamina $E$ y humanos no ha sido observado en todos los estudios y trabajos adicionales sobre la influencia de la dosis y de las especies debe ser llevados a cabo.

Los resultados de la presente investigación muestra que la administración de $\alpha$ tocoferol a conejos hipercolestrolémicos disminuye significativamente la oxidación de LDL a los dos días de su tratamiento y reduce el colesterol plasmático después de 
seis días de tratamiento, sugiriendo un potencial efecto benéfico en pacientes hipercolesterolémicos.

\section{REFERENCIAS BIBLIOGRÁFICAS}

1. AVIRAM, M. 1993.Modified forms of low density lipoproteins and atherosclerosis. Atherosclerosis. 98:1-9.

2. BOYD, H.; GOWN, A.; WOLFBAUER, G.; CHAIT, A. 1989.

Direct evidence for a protein recognized by monoclonal antibody against oxidatively modified LDL in atherosclerotic lesions, from Watanabe Heritable hyperlipidemic rabbits. Am J Pathol. 1989; 135:815-825.

3. BUCLEY, M.; GOA, KL.; PRICE, AH.; BROGDEN, EH. 1989. Probucol a reappraisal of its pharmacological properties and therapeutic use in hypercholesterolemia. Drugs. 37:761800.

4. BUEGE, JA.; AUST, SD. 1978. Microsomal lipid peroxidation. Methods of Enzimology. 52:302-310.

5. CAREW, TE.; SCKWENKE, DC.; $\begin{array}{lll}\text { STEINBERG, } & \text { D. }\end{array}$ Antiatherogenic effect of probucol unrelated to its hypercholesterolemic effect:evidence that antioxidants in vivo can selectively inhibit low density lipoprotein degradation in macrophagerich fatty streaks and slow the progression of atherosclerosis in watanabe heritable hyperlipidemic rabbit. Proc Natl Acad Sci USA. 84:7725-7729.

6. DAVIES, MJ.; WOOLF, N.1993. Atherosclerosis: What is it and why does it occur? Br Heart J. 69:S-3 -S-11.

7. DIEBER-ROTHENEDER, M.; PUHL, H.; WAEG, G.; STRIEGL, G.; ESTERBAUER, H. 1991. Effect of oral supplementation with $\mathrm{D}$-alpha tocopherol of the vitamin E content of human low density lipoprotein and resistance to oxidation. J Lipid Res. 32:1325-1332.

8. DUTHIE, G. 1991. Antioxidant hypothesis of cardiovascular disease. Trend Food Sci Techn. 1191:205-207.

9. ESTERBAUER, H.; JURGENS, G.; QUEHENBERGER, O. KOLLER. E.
1987. Antioxidation of human low density lipoprotein: Loss of polyunsaturated fatty acids and vitamin $\mathrm{E}$ and generation of aldehydes. J Lipid Res. 28:495-509.

10. ESTERBAUER, H.; STRIEGL, G.; PUHL, H.; OBERREITHER, S.; ROTHENEDER, M.; EL-SAADANI, M.; JURGENS, G. 1989. The role of vittamin $E$ and carotenoids in preventing the oxidation of low density lipoprotein. Annual New York Acad Sci. 570:254-267.

11. GEY, K.F; PUSKA, P.; JORDAN, P.; MOSER VK.1991 Inverse correlation between plasma vitamin $\mathrm{E}$ and mortality from ischemic heart disease in cross-cultural epidemiology. Am J Clin Nut. 53:326S-334S.

12. GODFRIED, SL.; COMBS, GF.; SAROKA, JM.; DILLINGHAM, CA.1989. Potentiation of atherosclerotic lesions in rabbits by a high dietary level of vitamin $\mathrm{E}$. $\mathrm{Br} \mathrm{J}$ Nutr. 61:607-614.

13. HABERLAND, M.E.; FONG, D. CHENG, L. 1988 Malondialdehydealtered protein occurs in atheroma of watanabe heritable hyperlipidemic rabbits. Science. 242:215-218.

14. HALLIWELL, B.; CHIRICO, S. 1993. Lipid peroxidation: its mechanism measurement and significance. Am J Clin Nutr. 57:715S724 S.

15. HAVEL, RJ.; EDER, HA; BRAGDON, JH. 1955. The distribution and chemical composition of ultracentrifugally separated lipoproteins in human serum. J Clin Invest. 34:1345-1353.

16. HEINECKE, JW.; SUZUKI, L.; ROSEN, H. 1987. The role of sulfurocontaining amino acids in superoxide production of low density lipoproteins modification of low density lipoproteins modification by arterial smooth muscle cells. J Biol Chem. 262:10098-10103.

17. HENEKENS, C.H. 1998. Antioxidant vitamins and cardiovascular disease: Current perspectives and future directions. European Heart Journal. 18:177-179 
18. HIROSE, M.; SHIBATA, M.; HAGIWARA, A.; IMAIDA, K.. ITO,

N. 1981. Chronic toxicity of butylated hydroxytoluene in wistar rats. Food Cosmet. Toxicol. 19:147-151.

19. INGOL, K.; WEBB, A.; WITTER, D.; BURTON, G.W. 1987. Vitamin e remains the major lipid soluble chain breaking antioxidant in human plasma livers in individuals suffereing from severe vitamin deficiency. Arch. Biochem. Biophys. 259:224-225.

20. JANERO, D. 1991. Therapeutic potential of vitamin $E$ in the pathogenesis of spontaneous atherosclerosis. Free Rad Biol Med. 11:129-144.

21. JIALAL, I. \& GRUNDY, S. 1992. Effect of dietary supplementation with alpha tocopherol on the oxidative modification of low density lipoprotein. J Lipid Res. 33:899-906.

22. JIALAL, I.; FULLER, C.; HUET, B. 1995. The effect of $\alpha$-tocopherol supplementation on LDL oxidation. Dose-response study. Arteriosclerosis Thromb Vas Biol. 15:190-198.

23. KANNEL, E.B.; CASTELLI, W.R.; GORDON, T.1979 Cholesterol in the prediction of atherosclerotic disease; new perspectives based on the Framingham study. Ann Intern Med. 90: 85-89.

24. KITA, T.; NAGANO, Y.; YOKODE, M.; ISCHII, K.; KUME, N.; OSHIMA, A.; YOSHIDA, H.; KAWAI, C. 1987. Probucol prevents the progression of atherosclerosis in Watanabe heritable hyperlipidemic rabbit, an animal model for familial hypercholesterolemia. Proc Natl Acad Sci USA.1. 84:5928-5931.

25. KLEINVELD, H.; DEMACKER, P.; STALENHOEF, A. 1998. Comparative study on the effect of low dose vitamin $E$ and probucol on the susceptibility of LDL to oxidation and the progression of atheroscleroses in watanabe heritable hyperlipidemic rabbits. Atherosclerosis Thromb. 14:1386-1391.

26. LOWRY, OH.; ROSEBROUGH, NJ.; FARR, AL.; RANDALL, AJ. 1951. Protein measurement with Folin phenol reagent. J Biol Chem. 193:265275.

27. MACLIN, L. 1998. VITAMIN E. In Handbook of Vitamins. Marcel Dekker, Inc. New York. 99-145.

28. PALINSKI, W. ROSEFELD, ME.; YIA-HERTTULA, S.; GURTNER, GC.; SOCHER, S.; BUTTER, SW.; PARTHASARATHY, S.; CAREW, T.E.; STEINBERG, D. WITZTUM, JL. 1989. Low density lipoprotein undergoes oxidative modification in vivo. Proc Natl Acad Sci USA. 86:1372-1376.

29. PARTHASARATHY, S. ; KHOO, JC.; MILLER, E.; BARNETT, J.; WITZTUM, J.; STEINBERG, D. 1990. Low density lipoprotein rich in oleic acid is protected against oxidative modifications: Implications for dietary prevention of atherosclerosis. Proc Natl Acad Sci USA. 87:3894-3898.

30. PRASAD,' K.; KALRA, J. 1993. Oxygen free radicals and hypercholesterolemic atherosclerosis. effect of vitamin E. Am Heart J. 125:958-973.

31. QIAO, Y.; YOOKOYAMA, M.; KAMEYAMA, K.; ASANO, G. 1993. Effect of vitamin $\mathrm{E}$ on vascular integrity in cholesterol-fed guinea pigs. Arteriosclerosis Thromb. 13:18851892.

32. QURESHI, A.; BURGER, W.; PETERSON, D.; ELSON, C. 1986. The structure of an inhibitor of cholesterol biosynthesis isolated from barley. J Biol Chem. 261:1054-1063.

33. RIBEIRO-JORGE, P.A.; CRUZ, L.; MICHICO, R; ALMEIDA, E. 1998. Improvement in the endotheliumdependent relaxation in hypercholesterolemic rabbits treated with vitamin E. Atheroscleoçrosis. 140:33-339.

34. RIEMERSNA, RA.; WOOD, DA.; MACINTYRE, C.; ELTON, RA.; GEY, K.F.; OLIVER, MF. 1991. Risk of angina pectoris and plasma concentrations of vitamin $\mathrm{A}, \mathrm{C}$ and $\mathrm{E}$ and carotene. Lancet. 337:1-5.

35. STEINBERG, D. PARTHASARATHY, $\quad$ S.; CAREW,TE.; KHOO, JC WITZTUM, JL. 1989. Beyond 
cholesterol: modification of low density lipoprotein that increase its atherogenicity. $\mathrm{N}$ Engl J Med. 320: 915-924.

36. STEINBRECHER, UP.; WITZTUM, JL.; PARTHASARATHY, S.; STEINBERG, D. 1987. Decrease in reactive aminogroups during oxidation or endothelial cell modification of LDL. Correlation with changes in receptor-mediated catabolism. Arteriosclerosis Thromb. 7:135-143.

37. TRABER, M.; KAYDEN, H. 1980. Alfa-tocopherol as compared with gamma-tocopherol is preferentially secreted in human lipoproteins.Ann $\mathrm{N}$ Y Acad Sci. 570:95-107.
38. WESTROPE, KL.; MILLER, RA.; WILSON, RB. 1982. Vitamin E in a rabbit model of endogenous hypercholesterolemia and atherosclerosis Nutr Rep Int. 25:83-88.

39. WILLIAMS, RJ.; MOTTERAM, JM.; SHARP CH.; GALLAGHER, PJ. 1992. Dietary vitamin $\mathrm{E}$ and the attenuation of early lesion developmente in modified watanabe rabbits. Atherosclerosis. 94:153-159.

40. WITZTUM, JL. 1993. Role of oxidised low density lipoprotein in atherogenesis. Br Heart J. 69:S12-S18. 


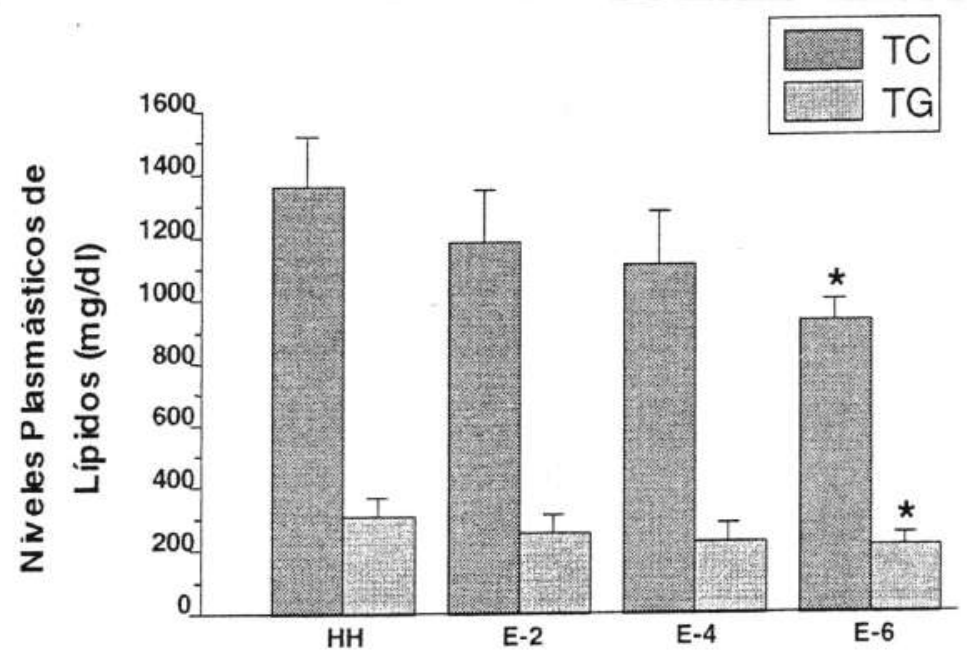

Figura 1. Niveles de Colesterol Total (TC) y Triaciglicéridos (TG) en el plasma de conejos hipercolesterolémicos (HH) y tratados con $\alpha$-tocoferol, durante los 2 (E-2), 4 (E-4) y 6 (E-6) días finales del experimento. Los valores son expresados como la media \pm desvío estándar Diferencia significativa en relación al grupo control se indica con asterisco $\left(^{*}\right)$ donde $\mathrm{p}<0.05$

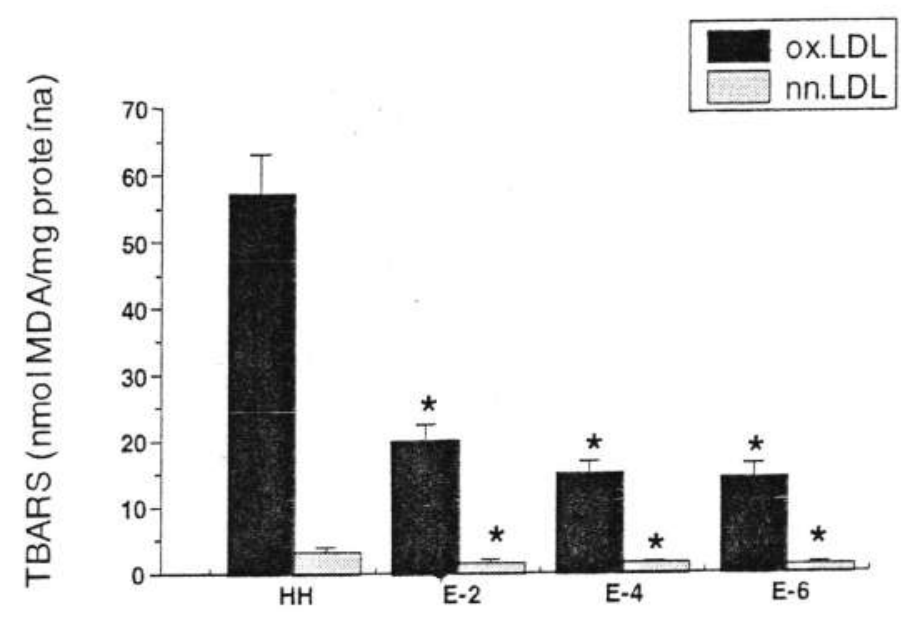

Figura 2 Concentración de Malondialdehído (MDA) en partículas de LDL nativa (nn-LDL) y LDL oxidada (ox-LDL) de conejos hipercolesteroémicos (HH) y tratados con $\alpha$-tocoferol, durante 2 (E-2), 4 (E-4) y 6 (E-6) días finales al experimento. Los valores son expresados como la media \pm desvío estándar. Diferencia significativa con relación al grupo control se indica con asterisco $(*)$ donde $\mathrm{p}<0.05$ 
Tabla 1 Niveles de Colesterol Total, (TC), Colesterol HDL (HDL), colesterol LDL (cLDL) y Colesterol VLDL (c-VLDL) en mg/dl, en plasma de conejos Hipercolesterolémicos, (HH) y tratados con $\alpha$-tocoferol, durante 2,4 y 6 días (E-2, E-4, E-6).

\begin{tabular}{lcccc}
\hline & HH & \multicolumn{1}{c}{ E-2 } & \multicolumn{1}{c}{ E-4 } & \multicolumn{1}{c}{ E-6 } \\
\hline TC & $1360.51 \pm 159.22$ & $1184.54 \pm 162.59$ & $1109.69 \pm 96.06$ & $933.16 \pm 65.98^{*}$ \\
c-LDL & $900.78 \pm 148.07$ & $791.24 \pm 115.61$ & $763.97 \pm 110.48$ & $651.26 \pm 71.03^{*}$ \\
c-VLDL & $410.53 \pm 42.50$ & $348.16 \pm 56.88$ & $290.79 \pm 51.73$ & $227.99 \pm 21.63^{*}$ \\
c-HDL & $49.20 \pm 4.63$ & $49.67 \pm 10.75$ & $49.88 \pm 5.08$ & $53.78 \pm 7.29$ \\
\hline
\end{tabular}

Los niveles se expresan en promedio \pm desvío estándar, donde $n=8$ ( $n$ representa el número de unidades experimentales por grupo) Diferencia significativa con relación al grupo control se indica con asterisco $(*)$ donde $\mathrm{p}<0.05$ )

Table 2 Composición (\% por peso) de la partícula de LDL

\begin{tabular}{lrccc}
\hline \multicolumn{1}{c}{$\%(w / w)$} & HH & E-2 & E-4 & E-6 \\
\hline Colesterol & $62.39 \pm 2.43$ & $61.27 \pm 1.29$ & $61.97 \pm 2.37$ & $60.87 \pm 1.72$ \\
Triacilglicéridos & $7.22 \pm 0.75$ & $7.39 \pm 0.77$ & $6.94 \pm 0.51$ & $6.95 \pm 0.56$ \\
Proteína & $20.38 \pm 1.47$ & $20.95 \pm 1.56$ & $20.53 \pm 1.82$ & $20.38 \pm 1.09$ \\
Fosfolípidos & $10.01 \pm 1.09$ & $10.39 \pm 0.76$ & $10.56 \pm 1.09$ & $11.80 \pm 0.67$ \\
\hline
\end{tabular}

Los valores se expresan en promedio \pm desvio estandar, donde $n=8$ ( $n$ representa el número de conejos estudiados por grupo) Diferencia significativa con relación al grupo control se indica con asterisco $\left(^{*}\right)$ donde $\mathrm{p}<0$. 